\title{
ALTENGLISCHES AUS SCHWEIZER HANDSCHRIFTEN.
}

\section{Alte Bibelglossen ans St. Gallen.}

\author{
Cod. SGall. 918. 8. Jahrhundert.
}

Die altenglischen Bibelglossen aus diesem berühmten Codex, der trotz oder vielmehr gerade wegen seiner kleinheit - er ist in duodez - zu den sehenswürdigkeiten der St. Gallener stiftsbibliothek gehört, hat zwar Steinmeyer bereits im vierten bande der Ahd. Glossen, s. 460, zum abdrucke gebracht und Kluge sie daraus in seinem ags. Lesebuch wiederholt. Ein erneuter abdruck in der Anglia wird aber trotzdem nicht unangebracht sein, weil es mir gelungen ist, die zahl der glossen um einige zu vermehren und den trefflichen druck Steinmeyers hier und da zu berichtigen. Um nur auf eins hinzuweisen: Nach Steinmeyer möchte es scheinen, als ob die hs. nur an einer einzigen stelle (Ahd. Gl. IV, 4602) die kapitale $R$, und zwar inmitten eines wortes, gebraucht. Unser druck wird zeigen, dafs sie sich anfangs, mitte und ende vorfindet. Ich halte mich in der wiedergabe genau an die hs., binde und trenne so wie sie und lasse die abkürzungen unaufgelöst, verzichte aber auf nachahmung des schriftzuges, der dem des Epinal ähnelt. Altenglisches hebe ich durch den druck hervor.

p. $139^{\circ}$ Cherogillus ani mal spino

7 S R $\left.\tilde{u}^{1}\right)$ maior quam hiricis griphẽ

1) St. bemerkt, vor $R$ sei $s$ zugefügt. $R$ ist vielmehr durchstrichen and $s$ zur korrektur an den rand geschrieben. 
p. $139^{8} \cdot \mathfrak{z}^{\mathfrak{l} z} \cdot$ aletum $^{2}$ ) similem ${ }^{\text {ut } 3)}$ aquilę

9 maioroauis ${ }^{4}$ ) tñ minor quam

10 ultor $^{5}$ ). garrula . hRoc . noctuam

p. $140^{1} \operatorname{nec}^{\left(\tau \operatorname{Rep}^{6}{ }^{6}\right)}$. bubonem $\left.{ }^{7}\right)$. ụuf $\left.{ }^{8}\right)$. laRum .

$\left.2 \mathfrak{m e u} \nmid \mathfrak{m e z}^{9}\right)$. mergulum niger.

3 auis mergit . subaquam pisces

4 quae rere id $\dot{\sim}$ dob fuzul ${ }^{10}$ ).

5 ibin a uis in africa ${ }^{11}$ ). habens.

6 longum Rostrum . cinnun $\left.{ }^{12}\right)$. $\mathfrak{f u}$

7 on . onoc Ratulum . auis q: sonitũ

$\checkmark$ facit inaqua RaRe dum lae $t$

9 felufoR $\left.{ }^{13}\right)$. porfinionem ${ }^{14}$ ). non fit

10 inbritania ${ }^{15}$ ). erodionem

p. $141^{1}$ ualuc haebuc ${ }^{16}$ ) chara drion . opu

2 pam . hupupa . uespertilionem ${ }^{17}$ ) quęl

2) = aëtum.

3) ut in dümuer, kleiner schrift nachtriiglich eingefügt; von St. übersehen.

4) o nachträglich über $a$, und zwar seitwärts ganz dicht herangesetzt; von St. übersehen.

s) $=$ uultur.

-) Der schreiber fand jedenfalls nectrebin vor.

$\left.{ }^{7}\right)$ Das erste $n$ anscheinend unterpunktiert, doch mag das klecks sein; von St. übersehen.

8) Ein starker und ein schwacher punkt unter dem ersten $u$; von St. übersehen.

9) Ein deutliches $i$ nach $g$; von St. übersehen.

10) Die vorlage hatte wohl dop fugul; vgl. anm. 6 .

11) St. versehentlich affrica.

12) Das erste $n$ durchstrichen, wie St. angibt.

13) Das wort gehört als übersetzung zum nächsten.

14) fi durchstrichen, was St. anzugeben verfehlt.

15) Das $t$ nach $i$ sehr dünn.

10) Deutlich als zwei wörter in der hs.; St. druckt sie als eins.

17) Das zweite $R$ durchstrichen, was St. nicht angibt. 
p. $141^{3}$ deRędę ${ }^{18}$ ) bruchus . similis $\dot{\sim}$

4 locús ${ }^{18}$ ) ${ }^{19}$ ) tñ maior attacus

5 ign̄o ${ }^{18}$ ) opi machus . igno.

6 locus ${ }^{18)}{ }^{20}$ ) ta. gRel hoppce

7 Reod . 18) 21) Corco dillus bestia

8 influmi ${ }^{18}$ ) ne similis lacęrtę ${ }^{22}$ )

9 id $\dot{\sim}$ adexan tñ maior $\dot{\sim}$ ita ut

10 homines manducat . migale igño

p. $142^{1}$ nisi similis $\dot{\sim}$ camelioni . camelion .

2 similis $\sim$ lacertę tñ sub aspectu

3 motat colores stelio bestia

4 inuenta $\sim$ similis ${ }^{18}$ ) $\quad \mathrm{s}^{23}$ ) lacerte

5 siffussa ${ }^{24}$ ) fuerit ${ }^{18}$ ) supn $^{n}$ en

6 aqua id $\sim$ inaqua ${ }^{18}$ ) ${ }^{25}$ ) labun

7 tur hęc uassa ${ }^{18}$ ) Alie

18) Hier beginnt ein ovales loch im pergament, das an all den mit 18) markierten stellen vorliegt.

19) St. druckt locus = locuste, das er so hätte auflösen müssen, da er ja die abkürzungen sonst auflöst, aber es steht deutlich locús; der abschreiber scheint den abkürzungsstrich seiner vorlage für einen Akzent angesehen zu haben. In der nächsten zeile druckt St. auch die abkürzung igno, die er nach seiner gepflogenheit hätte ignotıs auflösen müssen. Auf derselben zeile schreibt der kopist nachlässiger weise igno statt ignio = ignotus nach opi machus.

20) St. verfehlt locus ta als durch das loch im pergament getrennt anzugeben.

21) Gehört als übersetzung zu carectum.

$\left.{ }^{22}\right)$ St. druckt lacęrte.

2s) St. druckt $\&$, bezeichnet es aber als zweifelhaft; es ist ein deutliches durchstrichenes $s$; der schreiber hatte si zu schreiben begonnen, sah aber, dafs er lacerte ausgelassen hatte und strich $s$ aus.

24) = si fusa; St.'s vorschlag diffusa zu lesen, parst nicht in den zusammenhang; denn der erklärer will sagen: wenn man wasser über das tier in einem wasserbehälter giefst, so zerfallen diese gefälse. aquario.

${ }^{25}$ ) Der schreiber hat den abkürzungsstrich vergessen; lies $a \overline{q u a}=$ 
p. $142^{8}$ tum muleri $\left.{ }^{18}\right)^{26}$ ) modicus ${ }^{26}$ )

9 miluü z̆lda uultor modico. ma

10 ior quam aqua ${ }^{27}$ ) 7 p . c. milia . senti

p. $143^{1}$ potest cadaues ${ }^{28}$ ) laRũ hR $\propto z^{R} \propto$

2 adrianus $d \tilde{t}$ meum ${ }^{29}$ ) ề ibinen ${ }^{30}$ )

3 id $\sim$ fcReb . quimittit aquam deore

4 suo incuculum ${ }^{31}$ ) suũ utpossit de

5 gerere indeq: me dici ipsam ar

6 tem dedicerunt ${ }^{32}$ ) Onoc Ratulum

7 qsi anata $\tilde{n}$ eadem $\dot{\sim}$ tñ nec nos

8 habemus. Chara drion . 7 ipsam

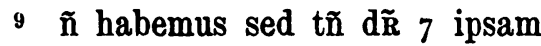

10 uolare prmedias noctes insub

11 limitatę cęli . por phirionem dr̃

p. $144^{1}$ qđ ipsa inlibia sit esseq: auiũ

2 pulcherrima pene ideo q: eã

3 uolunt Reges habeke indomib:

4 suis . sepissime Migale modi

5 cus $\tilde{q}$ si $\left.\operatorname{ca\tau } \tau e^{33}\right)$. stelio minor $\dot{\sim}$ quã

museri
${ }^{28}$ ) In der urvorlage dürfte soricarius modicus gestanden haben, das zum vorliegenden im laufe der abschriften zusammenschrumpfte; ae. muisere 'mänseadler' (Kluge) ist in denWtbb. nachzutragen.

27) Der abschreiber hat hier wieder den abkürzungsstrich vergessen; lies $a \overline{q u a}=a q u i l a$ (so St.).

28) Versehen für cadauer.

29) Ist natürlich das latinisierte ae. meu.

so) Versehen für ibin.

81) St. verfehlt das zeichen über dem ersten $c$ anzugeben; es ist vielleicht als tilgungszeichen für cu beabsichtigt, also cuculum zu culum karrigiert, wie St. will.

82) So!

8s) Ursprünglich dürfte catta gestanden haben. 
6 lacerta id $\dot{\sim}$ cdexe naldeq:

i uenenosa omnem q: parietẽ

8 penetrat licet lapidiũ $\left.{ }^{34}\right)$ ñresis

9 tit. Chero gillũ 7 hinix . unum

10 st pene inomni similitudine

11 ut porcus . nisi qd minores sit

p. $145^{1}$ quam porci sedtamen longe

2 statura ${ }^{35}$ ) $\left.\operatorname{sint}{ }^{36}\right) 7^{37}$ ) inmonte sinai

3 inscissuris petras ${ }^{38}$ ) maxime

4 habundant:

Ganz entgangen sind Steinmeyer folgende Bibel-glossen in demselben Codex:

p. $105^{\prime}$ philosi $^{1}$ ) siluestri $\left.{ }^{1}\right)$ homines ululę Jesai. 1321.22

2 genera auium sikinę ${ }^{2}$ ) monstra q:

3 dam tribular ${ }^{3}$ ) ez̃de 7 trachas $^{3}$ ) d $\tilde{\mathbf{k}} 1$ Paralip. $20^{3}$

$4 \stackrel{\nabla}{{ }^{*}}$ ezaentarceutina $\left.^{4}\right)$ ppum ${ }^{5}$ ) nomen lig 2 Paralip. $2^{8}$

5 ni . habens funem depalme rus

6 cam .i. Rindı:- issiodonus delitteris: : 6)

Zeile 3: eqgde, das zeichen über dem $g$ dürfte dieselbe geltung haben wie das $v$ über $a$, d. h. $d$, von egae und vernacule bedeuten. $\mathrm{Zu}$ egde 'tribula' vgl. egdae 'erpica' im Erfurter und Corpusglossar. Dals auf z. 4 statt des überlieferten egae auch wieder egde zu lesen ist, beweist traha eipe (verdruckt cipe) in Elfrics Vocabular, WW. 105². Der schreiber dürfte an lat. egent arceuthina gedacht haben.
34) sit ist ausgefallen.
3s) Wohl für staturae.
s6) Versehen für sunt.
37) Von St. übersehen.
38) Versehen für petranx = petrarum.
1) So!
2) Die Vulgata hat sirenes.
3) So! für tribulas und trahas.
4) d. h. egde . et arcentina (= et arceuthina).
s) Natürlich $=$ proprium.
в) Auszüge aus dieser schrift des Isidor folgen. 
Z. 5-6: Die glosse scheint auf einer vorlage zu beruhen, ruscam .i. rindr

wo stand: habens funem de palmae cortice(m) ; woher sie stammt, habe ich nicht ermittelt; zu rusca vgl. ir. vusc 'rinde', 'korb'. Stokes-Bezzenberger halten das ir. wort für Entlehnung aus dem Germanischen, auf mhd. rusche 'binde', engl. rush verweisend.

\section{Bedas Sterbegesang.}

Aus Cod. SGall. 254. 9. jahrhundert.

Dieses wichtige nordhumbrische fragment ist zwar auch schon veröffentlicht worden, so von Sweet, OET., p. 140, Kluge und von Schipper in seinem ae.-me. Lesebuche, aber die veröffentlichungen sind nicht ganz diplomatisch genau. Ich glaube den interessen der Anglistik zu dienen, wenn ich im folgenden den genauen handschriftlichen text gebe. Er findet sich auf s. 253 als teil eines stückes, das s. $252^{9}$ beginnt und betitelt ist:

INCIPIT DE UALITUDINE ET OBITU UENERABILIS BEDA ${ }^{1}$ ) P $\widetilde{R I}$.

Dann heifst es auf s. 253:

Zeile 3 amonebat et in n⿳亠丷a quoq: lingua

4 ut erat doctus innostris carminib;

5 dicens detibili exitu animarum

6 ecorpore Fore the neid faerae na

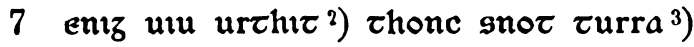

8 Than him thar ste toymb hỳcggan

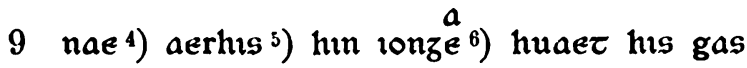

10 tae godaes a\&h thai) yflaes aefer

11 deoth daege doe mid uueor thes)

1) So! 2) So! Kluge niuuiurthit, versehen für niuurthit. Diese lesung wenigstens möchte ich empfehlen. 3) So! $\quad$ 4) So! 5) So! o) So! r) So! r) So! 